\title{
Performance Assessmentfor Hyper Pure Germanium (HPGe) and Gallium Arsenide (GaAs) detectorsin NDA Techniquesusing MCNP code
}

\author{
R.A.El-Tayebany* \\ Department of Nuclear Safeguards and Physical Protection, Egyptian \& radiological \\ regulatory Authority (ENNRA) \\ 3 Ahmad El-Zomor Str., El-Zohoor Dist., Nasr City, P.O. Code 11762, Cairo, Egypt
}

\begin{abstract}
Development of equipment and techniques for safeguards applications is necessary that assisted in keeping pace with the evolution of new technology. The independent verification measurements of nuclear material using a variety of Non-Destructive Assay (NDA) instrumentation is main target in safeguards field. The paper presents comparative study for Gamma radiation detectors characteristics that are simulated by MCNP code. The detection system studied different energy lines which were considered as the significant in safeguards measurements to calculate the pulse height distribution using tally (F8). Hyper Pure Germanium (HPGe) and Gallium Arsenide (GaAs) detectors were simulated and proposed for semiconductor detectors. The results were compared and interpreted.
\end{abstract}

Keywords:Safeguards, MCNP Code, Gamma radiation detectors, Non-Destructive Assay (NDA).

*TEL.: +201016060637

Email: reltyebany@ymail.com

\section{Introduction}

The process of safeguards technology development, deployment, and use should be carried out in such a way that it supports the safeguards verification mission, while also being efficient in terms of both cost and time.

This process generally begins with research and development to progress the technology to a point that it may be used or installed within a nuclear facility. The equipment developer may be unable to directly communicate interact with safeguards inspectors, who are the targeted end users of the technology. As a result, an equipment developer may not receive input on how best to design equipment such that it optimizes inspectors' activities through improvements in measurement efficiency and/or accuracy, ergonomics, portability, and so on, or feedback on previously designed equipment [4].

The sensitivity of semiconductor detector which used to detect single gamma-quanta (discrete sensitivity) isdefined as ratio of pulse number $\mathrm{N}$ to unit of radiation dose. Energy dependence of cross-sections of gammaquantuminteraction with detector material is a basic factor which defines the behavior of sensitivity with changeof gamma-quantum energy. The total attenuation coefficient of gamma-quantum flowin the semiconductors ischanged on four orders of magnitude in the energy range between $10 \mathrm{keV}$ and $1 \mathrm{MeV}$ [1]. 


\section{Detectors}

\subsubsection{HPGe detector}

By far the most common type of application for Ge detectors is basic gamma-ray spectroscopy, where a single detector is used with a single channel of electronics to provide energy information. With modern low-noise electronics and Ge materials, the best energy resolution achieved with Ge detectors at gamma-ray energies is close to the fundamental limits imposed by charge generation statistics. High Purity Germanium (HPGe) gamma-ray detectors have been widely used in the nuclear and industrial sectors for many applications over many years. The advantages of HPGe detectors in terms of energy resolution, efficiency, sensitivity, and ease of spectrum analysis have been clear in a wide range of applications both fixed-installed and in situ.

\section{GaAs detectors}

Gallium Arsenide (GaAs) is the only semiconductor detector that operated in room temperaturewith high electronmobility approximately $7000 \mathrm{~cm} 2 \mathrm{~V}^{-1} \mathrm{~s}^{-2}$ andwide band gap with value $1.4 \mathrm{eV}$. A combination of these twocharacteristics leads to potential highperformance gammaspectrometry. The main limitationimposed on this detector technology is the difficulty inobtaining spectrometry-grade bulk GaAs material. Inaddition, due to their poor resistivity, only thin detectorscan perform spectrometric measurements. This limits theapplication of such detectors to low energy measurements.However, the possibility of a technological breakthroughmight come from the development of ternary material, which might solve the problem of resistivity. Currentprojects are researching the possibility of cooling GaAsdetectors using the Peltier Effect to achieve higherresistivity [2]. Its density $5.3176 \mathrm{~g} \mathrm{~cm}-3$. The wide band gaps of these compounds mean that they have high resistivity $(>109 \Omega \mathrm{cm})$ and are capable of room temperature operation.

The thickness of the crystals ranged from $38 \mu \mathrm{m}$ to $120 \mu \mathrm{m}$. Au-GaAs surface barrier detector was made of epitaxial crystal. Some of the detector were encapsulated in a can with a 50 $\mu \mathrm{mBe}$ window by welding a can to the detector holder. The detector with high energy resolution and good charge collecting characteristics was selected by alpha spectrometry at room temperature. Energy resolution of the detector for gamma-rays up to about $200 \mathrm{keV}$ was very good at room temperature operation. The best energy resolutions taken with a GaAs detector were $3 \mathrm{keV}$ (fwhm) and $3.8 \mathrm{keV}$ for $241 \mathrm{Am} 59.6 \mathrm{keV}$ and $57 \mathrm{Co} 122 \mathrm{keV}$, respectively, at room temperature. In order to study the applicability of the detector for nuclear safeguards, the measurements of ${ }^{235} \mathrm{U}$ gamma-ray spectrum have been carried out at room temperature. It was clarified that the gamma-ray spectrum of enriched U sample could be measured in high resolution with GaAs detector at room temperature, and that the content of ${ }^{235} \mathrm{U}$ in enriched $\mathrm{U}$ sources could be determined by measuring gamma-ray spectrum with GaAs detector.However, gamma-ray counting efficiency of the detector was not enough to built portable type instrument of gamma-ray spectrometer used for routine works of nuclear safeguards. In order to improve gamma-ray counting efficiency of the detector, doubleepitaxial-layer detector has been studied. The preliminary results showed that the improvement of the detector gamma-ray counting efficiency was possible by using doubleepitaxial-layer structure. It was also clarified that the good quality GaAs crystal was a key to obtaining a low noise, good charge collection detector [7]

The low intrinsic carrier density of GaAs in a pure (undoped) form indicates that GaAs is intrinsically a very poor conductor and is commonly referred to as being semi-insulating. This property is usually altered by adding dopants of either the $\mathrm{p}$ - (positive) or $\mathrm{n}$ - (negative) type. 


\section{MCNP}

The MCNP is an advanced MC simulation program containing all necessary cross-section data for neutron, photon and electron transport calculations. Monte Carlo simulation is considered as a sequence of random numbers which occurs during the simulation. When repeating the sequence of random numbers, the simulation will give results that agree with those gotten from the first sequence to within some 'statistical error'. The General Monte Carlo Code (MCNP-5) was used to compute the absolute efficiency of detector [9].The characteristics and specifications of the planar HPGe detector and used samples were modeled as much details as possible in order to simulate the experimental setup. The number of created input files that run for this calculation were 6 input files. The histories used in the created input files were 108 histories (number of photons) with run time 20 minutes. The specifications of the used laptop is $2.5 \mathrm{GHz}$ Intel Core i5 processor, since a tally F8 is used to determine pulse height of the detector. The absolute efficiency of the detector at different energy lines were calculated by means of that tally.

The MCNP-5 code has been used for modeling thedetector response, since it contains a tally, F8, which isspecific for detector pulse height determination. Thefraction of gamma-rayswith certain energy - absorbedin the detector active volume represents its absolute fullenergy peak efficiency at that energy [8].

\section{Detector simulation}

The measuring system used in the experimental work includes High Purity Germanium detector (HPGe) with model Canberra GL0515R and active area of $540 \mathrm{~mm} 2$. The height is $1.5 \mathrm{~cm}$ and FWHM at $122 \mathrm{keV}$ is $540 \mathrm{eV}$.Fig.(1) shows that the internal layers of the simulated detector.

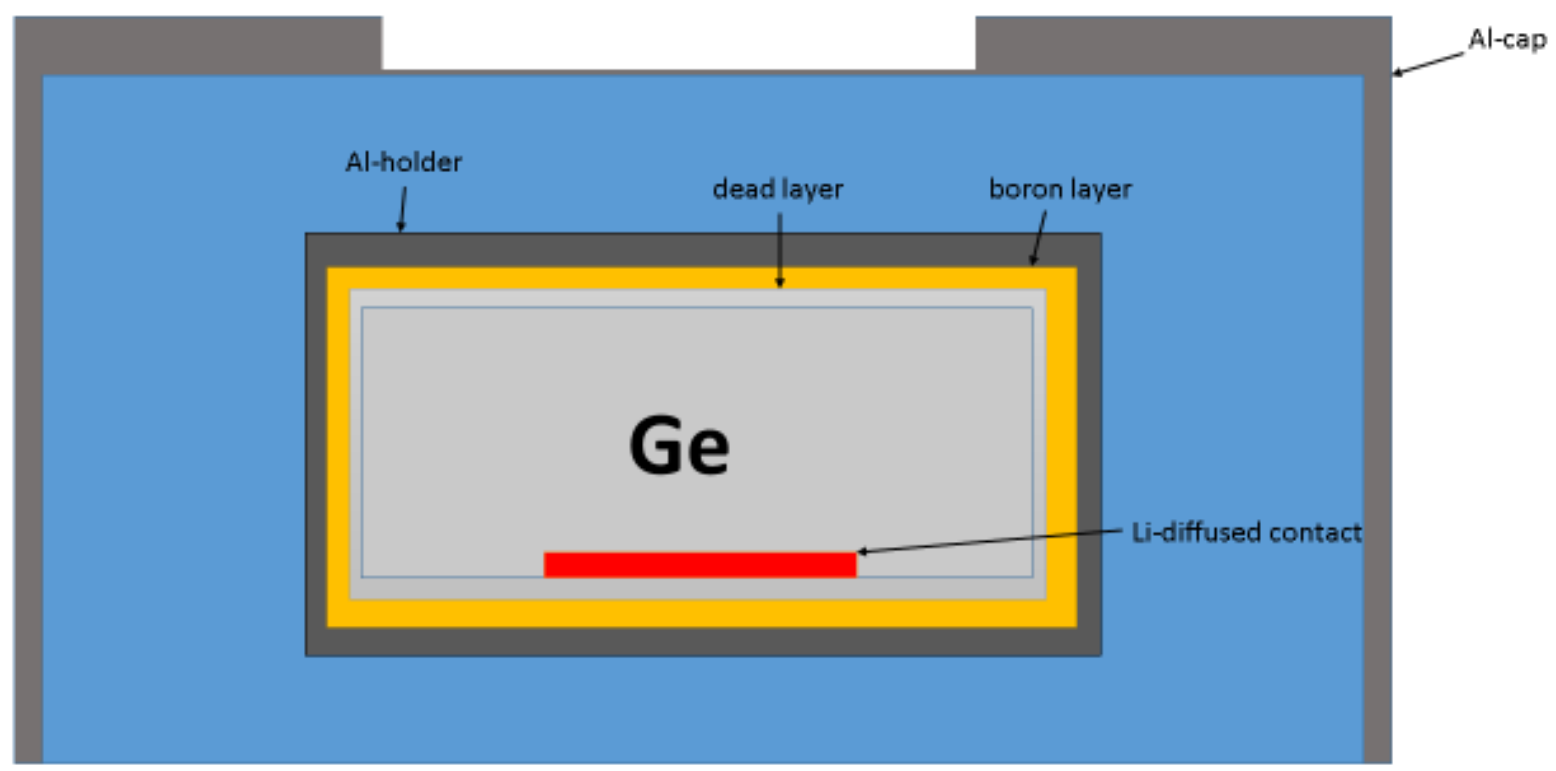

Fig(1). Internal structure of HPGe detector

\section{Results}

The input files are executed to obtain pulse height distribution by tally(F8) with changing in history that is defined as the number of incident photons on the detector surface. The number of history is taken to be in range 102 to 107 as shown in table (1).49 input files were created to obtain table (1). Each file was run from 15-35 minutes. The input file described the measuring system which consist of the simulated detector and point source that was located 
in front of the detector face. The choice of energy lines values was depend on detector performing in wide range of energy to cover all the safeguards application.

Table(1). History impact (nps) on pulse height distribution in MCNP code

\begin{tabular}{llllll}
\hline & Co-57at & U-235 at & cs-137 at & Co-60 at & Co-60 at \\
& $122 \mathrm{Kev}$ & $185.7 \mathrm{Kev}$ & $661.7 \mathrm{Kev}$ & $1173.2 \mathrm{Kev}$ & $1332.5 \mathrm{Ke}$ \\
History & & & & & v \\
\cline { 5 - 6 }
\end{tabular}

Pulse height distribution

\begin{tabular}{llllll}
\hline $\mathbf{1 0}^{2}$ & $1.40 \mathrm{E}-01$ & $9.00 \mathrm{E}-02$ & $7.90 \mathrm{E}-3$ & $1.00 \mathrm{E}-02$ & $1.00 \mathrm{E}-02$ \\
\hline $\mathbf{1 0}^{3}$ & $1.63 \mathrm{E}-01$ & $8.80 \mathrm{E}-02$ & $8.00 \mathrm{E}-03$ & $8.00 \mathrm{E}-03$ & $6.00 \mathrm{E}-03$ \\
\hline $\mathbf{1 0}^{4}$ & $1.51 \mathrm{E}-01$ & $8.65 \mathrm{E}-02$ & $1.17 \mathrm{E}-02$ & $6.40 \mathrm{E}-03$ & $4.40 \mathrm{E}-03$ \\
\hline $\mathbf{1 0}^{5}$ & $1.50 \mathrm{E}-01$ & $8.45 \mathrm{E}-02$ & $1.02 \mathrm{E}-02$ & $4.57 \mathrm{E}-03$ & $4.20 \mathrm{E}-03$ \\
\hline $\mathbf{1 0}^{6}$ & $1.48 \mathrm{E}-01$ & $8.36 \mathrm{E}-02$ & $9.75 \mathrm{E}-03$ & $4.59 \mathrm{E}-03$ & $4.00 \mathrm{E}-03$ \\
\hline $\mathbf{1 0}^{7}$ & $1.49 \mathrm{E}-01$ & $8.40 \mathrm{E}-02$ & $9.81 \mathrm{E}-03$ & $4.59 \mathrm{E}-03$ & $3.97 \mathrm{E}-03$ \\
\hline
\end{tabular}

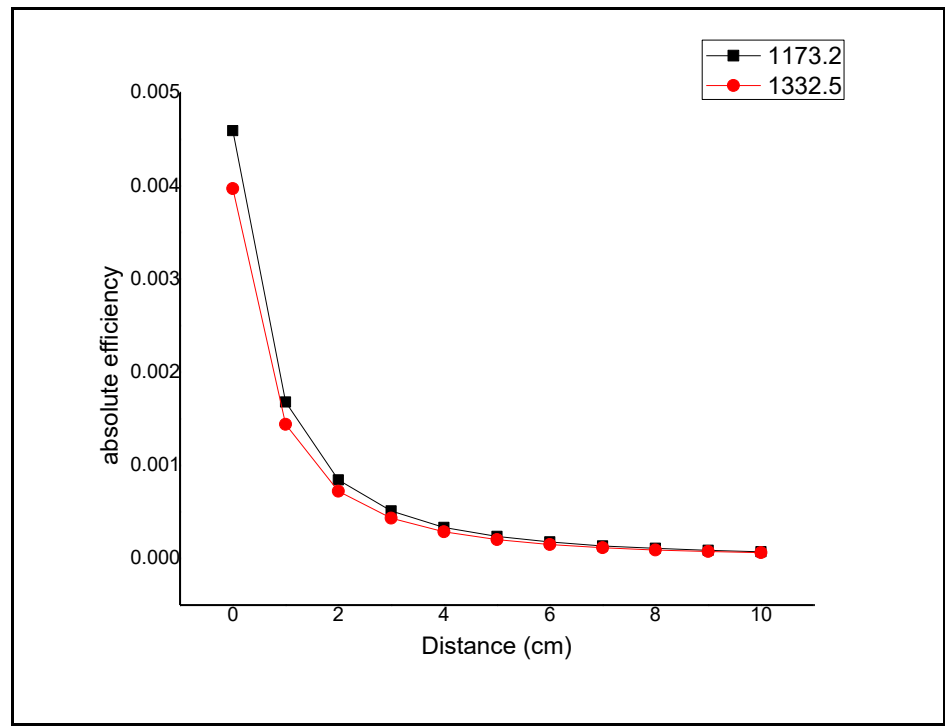

Fig.(2) S-D distance change versus Absolut efficiency at higher energy for HPGe detector by MCNP-5

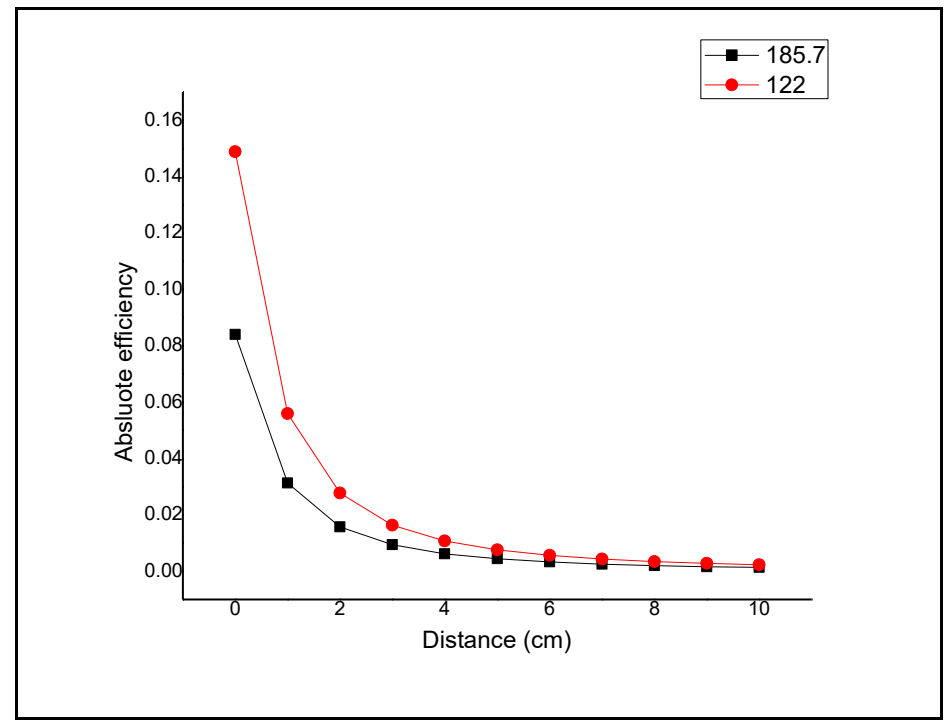

Fig.(3) S-D distance change versus Absolut efficiency at lower energy for HPGe detector by MCNP-5 
At GaAs detector simulation, the ratio of gallium atoms to arsenic atoms must be known in MCNP simulation. So that, different ratios were assumed to reach at ideal ratio at which the detector efficiency is high as possible.Table (2) described various values of Ga: As ratios at different energy lines.

Table (2). The effect of Ga :As ratio on the pulse height distribution at various energy lines

\begin{tabular}{|c|c|c|c|c|c|}
\hline Energy lines (Kev) & $\mathbf{1 : 1}$ & $\mathbf{1 : 2}$ & $\mathbf{2 : 1}$ & $\mathbf{1 : 9}$ & $\mathbf{9 : 1}$ \\
\cline { 2 - 6 } & \multicolumn{5}{|c|}{ Pulse height distribution } \\
\hline $\mathbf{1 2 2}$ & $1.49 \times 10^{-1}$ & $1.51 \times 10^{-1}$ & $1.48 \times 10^{-1}$ & $1.53 \times 10^{-1}$ & $1.45 \times 10^{-1}$ \\
\hline $\mathbf{1 8 5 . 7}$ & $8.44 \times 10^{-2}$ & $8.60 \times 10^{-2}$ & $8.27 \times 10^{-2}$ & $8.81 \times 10^{-2}$ & $8.04 \times 10^{-2}$ \\
\hline $\mathbf{6 6 1 . 7}$ & $9.94 \times 10^{-3}$ & $1.02 \times 10^{-2}$ & $9.70 \times 10^{-3}$ & $1.05 \times 10^{-2}$ & $9.38 \times 10^{-3}$ \\
\hline $\mathbf{1 0 0 1 . 2}$ & $5.73 \times 10^{-3}$ & $5.85 \times 10^{-3}$ & $5.60 \times 10^{-3}$ & $6.02 \times 10^{-3}$ & $5.42 \times 10^{-3}$ \\
\hline $\mathbf{1 3 3 2 . 5}$ & $4.03 \times 10^{-3}$ & $4.13 \times 10^{-3}$ & $3.94 \times 10^{-3}$ & $4.25 \times 10^{-3}$ & $3.81 \times 10^{-3}$ \\
\hline
\end{tabular}

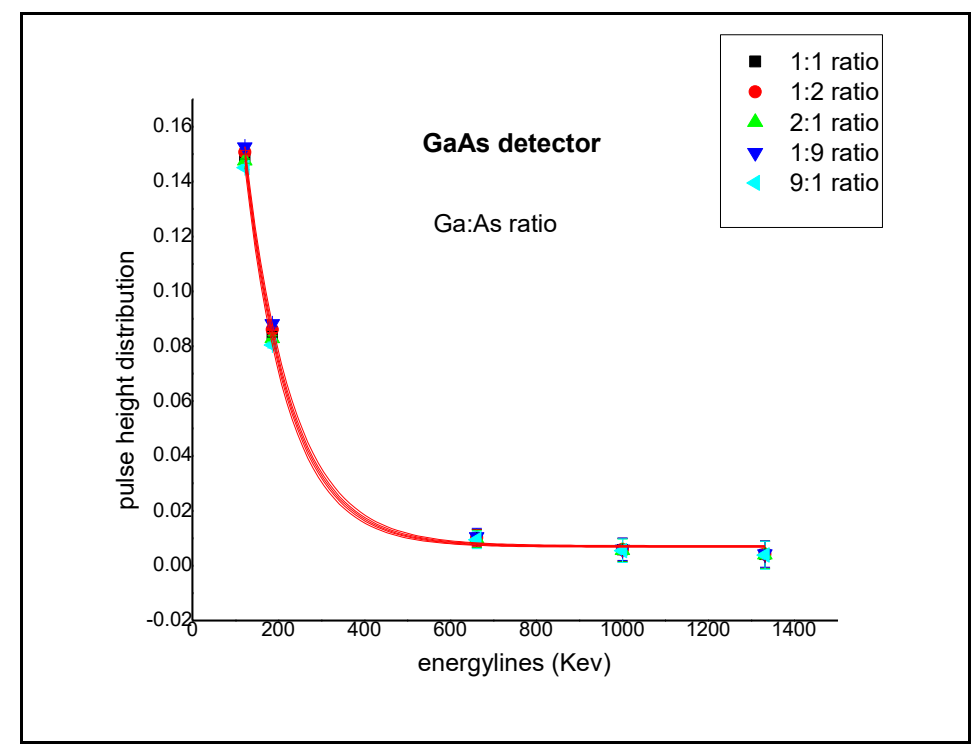

Fig.(4) pulse height distribution changes with the energy lines in case of Ga :As ratios A slight difference in the resultsof pulse height distribution with $\mathrm{Ga}$ : As ratios as shown inFig.(4). The curve is fitted by exponential function which is given by eq.(1) and fitting parameters of each Ga: As ratio curve are shown in table (3).

$$
Y=A_{1} e^{-x / t}+Y_{0}
$$

Table (3). Fitting parameters of eq.(1)

\begin{tabular}{c|ccc} 
Description & $\boldsymbol{y 0}$ & $\boldsymbol{A 1}$ & $\boldsymbol{t}$ \\
\hline $\mathbf{1 : 1}$ ratio & 0.00702 & 0.45565 & 104.71964 \\
$\mathbf{1 : 2}$ ratio & 0.00717 & 0.45216 & 106.29447 \\
$\mathbf{2 : 1}$ ratio & 0.00689 & 0.45854 & 103.22512 \\
$\mathbf{1 : 9}$ ratio & 0.00731 & 0.44649 & 108.66734 \\
$\mathbf{9 : 1}$ ratio & 0.00674 & 0.46216 & 101.15828
\end{tabular}

The previous results showed that although there were slightly differences of the pulse height distribution values but, the preferred ratio for Ga: As ratio was 1:9. 
As a result to that, comparative study is performed between the HPGe detector and GaAs detector where, the last one takes the same dimensions as the first one at that selected ratio. The results are presented as in Fig. (5).

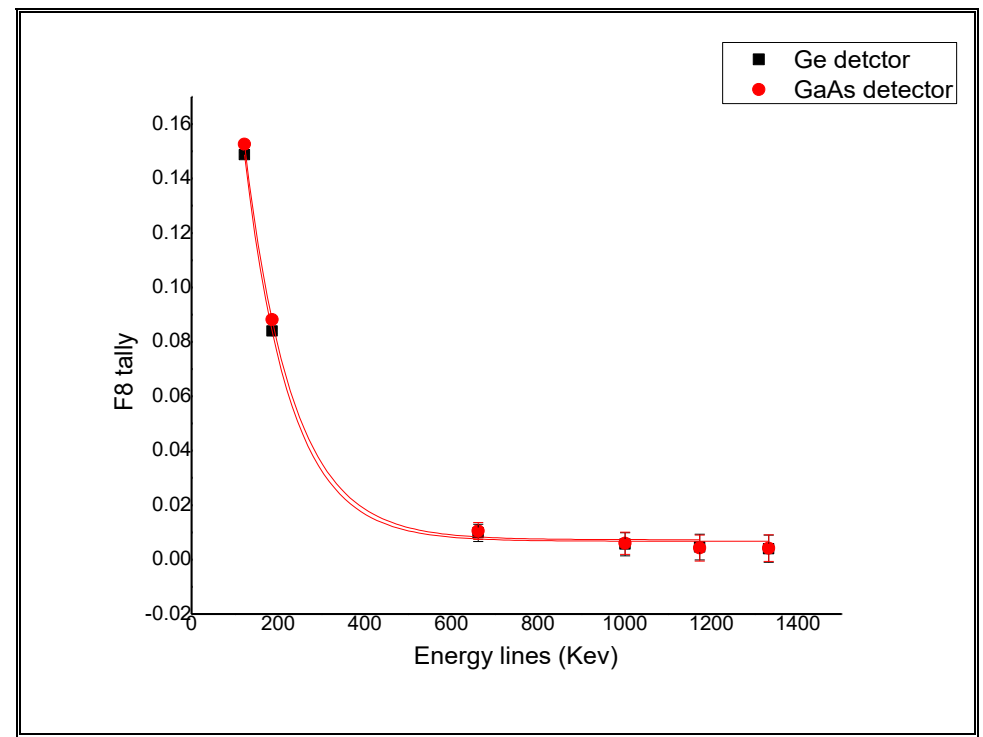

Fig.(5) comparative results using F8 tally calculations between the HPGe detector and GaAs detector

\section{Conclusion}

Performance Assessment fortypes of Gamma ray detectors was the main targetfor this work. The pulse height distribution was calculated by MCNP-5 code using tally (F8) for the presented detectors. Tally (f8) calculated the pulse height distributionat different values of energy lines along wide range of energy scale to show the performance of each used detector in lower and higher energy range. The simulated results proved that $\mathrm{HpGe}$ detector gave the best indication for pulse height distribution along the energy range of measurements. Although, GaAs semiconductor detector is operated at room temperature. The simulated calculations were performed for different values of $\mathrm{Ga}$ : As ratios. The results proved that although there were slightly differences of the pulse height distribution values but, the preferred ratio for Ga: As ratio was 1:9. The simulated GaAs detector gave the best results only in low energy range of gamma radiation detection. So that, the development of GaAs may be achieve results consistent with the requirements of safeguards applications under certain conditions. First, its crystal should be treated to be thicker to cover all the significant energy line that measured during safeguards application, as well as, The Physical strength of Gallium arsenide single crystals are very brittle. For this reason, the detector couldn't use in safeguards routine work until increasing its hardness and safety to avoid any damage during safeguards inspection.

\section{References}

1- A.A. Zakharchenko, DETERMINATION OF SENSITIVITY OF SEMICONDUCTOR DETECTORS OF GAMMA-RADIATION, PACS29.40.Wk, 85.30De, 07.85.-m.

2- R. Carchon, M. Moeslinger, L. Bourva, C. Bass, M. Zendel, Gamma radiation detectors for safeguards applications, Nuclear Instruments and Methods in Physics Research A 579 (2007) 380-383. 
3- P. N. Luke1, M. Amman1, C. Tindall1, J. S. Lee1, Recent developments in semiconductor gamma-ray detectors, 1Lawrence Berkeley National Laboratory, Berkeley, CA 94720, USA.

4- Alicia Swift1, Brian Paul2, Karen Hogue1, Bruce Moran1, Jill Cooley1, Design Considerations for the Field Use of Safeguards Technology, Y-12 National Security Complex, Oak Ridge, TN, USA.

5- Douglas S. McGregor, Materials for Gamma-RaySpectrometers: InorganicScintillators, Annual Review of Materials Research, April 4, 2018.

6- Berkeley Nucleonics Corporation - 2955 Kerner Blvd - San Rafael CA 9490.

7- Kobayashi, T.; Kuru, I. DEVELOPMENT OF GALLIUM ARSENIDE GAMMA SPECTROMETRIC DETECTOR, (Tokyo Shibaura Electric Co. Ltd., Kawasaki, Kanagawa (Japan). Research and Development Center), International Atomic Energy Agency, Vienna (Austria).

8- W. El-Gammal, Verification of $235 \mathrm{U}$ mass content in nuclear fuel platesby an absolute method, Nuclear Instruments and Methods in Physics Research A 570 (2007) 446-453.

9- D. P. Landau and K. BINDER, A Guide to Monte Carlo Simulations in Statistical Physics, 3rd ed., New York: Cambridge University Press, xv, 471 pp., ISBN: 978-0-52176848-1, 2009. 